\title{
10 Die Männer des 2. Weltkriegs: Das Trauma des „starken Geschlechts“
}

\author{
Svenja Eichhorn und Elmar Brähler
}

Im Kapitel zum „Alternden Mann“ des „Ersten Deutschen Männergesundheitsberichts“ wird das Label des "starken Geschlechts" kritisch betrachtet und als landläufiges Vorurteil beschrieben (Seikowski u. Paasch 2010). Auch innerhalb des vorliegenden Textes werden am Beispiel der Männer, die den 2. Weltkrieg (WK II) miterlebten, die kollektiven Folgen der Erlebnisse übersichtsartig betrachtet und damit versucht, das Leid der Männer anzuerkennen, die auch „schwaches Geschlecht" sein dürfen.

Folgender Beitrag betrachtet das Mann-Sein mit der Erfahrung einer Kriegsteilnahme am Beispiel des 2. Weltkriegs aus heutiger Sicht. Der Fokus wird hierbei auf dem Aspekt der Traumatisierung und besonders der Posttraumatischen Belastungsstörung (PTBS) liegen. Hierbei ist zu berücksichtigen, dass Forschungsergebnisse zu psychischen und körperlichen Folgen einer Kriegsteilnahme in jedem Falle auf einer Selektion der teilnehmenden Stichproben basieren, denn ein Großteil der Kriegsteilnehmer ist gefallen oder vorzeitig aufgrund der erhöhten Belastungssymptomatik und einhergehender körperlicher Symptome verstorben (Pizarro et al. 2006). Hierbei spielt auch die erhöhte Suizidrate bei Kriegsüberlebenden eine Rolle (Kühn 2009; Maguen et al. 2011).

\subsection{Folgen der Kriegstraumatisierung}

\subsubsection{Posttraumatische Belastungsstörung}

Ob militärische oder zivile Beteiligung, ob als Kind, Jugendlicher oder als Erwachsener, das Miterleben des 2. Weltkriegs dürfte in jedem Falle Spuren bei den Teilnehmenden hinterlassen haben.

Studien zeigen vielfach, dass Männer sowohl innerhalb eines Kriegs, als auch im Alltag mit größerer Wahrscheinlichkeit mehr potenziell traumatisierende Ereignisse erleben, als Frauen (Fairbank et al. 2000; Spitzer et al. 2008). Insgesamt birgt Kriegserleben eine mehrfach erhöhte Wahrscheinlichkeit der Traumatisierung (Brähler et al. 2004). Auch andere psychopathologische Symptome wie beispielsweise Angst oder Depression können durch Vertreibung (vgl. Beutel et al. 2007; Kuwert et al. 2009), das Aufwachsen ohne den Vater (vgl. Franz et al. 1999; Decker et al. 2005; Heuft 2001) 
oder direktes Erleben von Kampfhandlungen (vgl. Heuft et al. 2007) auftreten und zum Teil über Jahrzehnte andauern.

Auch wenn Männer im Vergleich zu Frauen insgesamt weniger häufig psychopathologische Symptome oder Traumafolgestörungen wie die Posttraumatische Belastungsstörung ausbilden (Breslau 2009), muss davon ausgegangen werden, dass die Gruppe der heute alten Männer, die den 2. Weltkrieg miterlebten, eine höhere Psychopathologie aufweist, als Männer ohne diese Erfahrung. Die Gruppe schließt ehemalige Kriegskinder, Kindersoldaten und „erwachsene“ Soldaten mit ein (vgl. Kuwert 2006; Forstmeier 2009).

Maercker und Kollegen (2008) zeigen anhand einer Stichprobe der Allgemeinbevölkerung eine deutlich erhöhte PTBS-Prävalenz bei über 6o-jährigen Deutschen. Diese Altersgruppe wies eine deutlich höhere Häufigkeit der Störung auf als die anderen untersuchten Kohorten (vgl. Glaesmer et al. 2010). Im Geschlechtervergleich finden sich keine Unterschiede hinsichtlich des Vorhandenseins einer PTBS im Alter (bei über 65-Jährigen) (Spitzer et al. 2008), was möglicherweise daher rührt, dass sich eine höhere Traumaexposition bei Männern mit der größeren Wahrscheinlichkeit der Entwicklung einer PTBS bei Frauen ausgleicht und die PTBS-Prävalenzen hier, nach Kriegserleben, korrespondieren. Dieses Ergebnis zeigt die Vulnerabilität der „WK IIMänner" bis ins Alter, wo diese doch im Allgemeinen weniger häufig an psychischen Symptomen wie Depression oder Angststörungen leiden als Frauen. In einer neueren Untersuchung wurde dies erneut belegt, wobei aufseiten der Männer in Zusammenhang mit verschiedenen psychischen Beeinträchtigungen stärkere Einschränkungen im alltäglichen „Funktionieren“ und im sozialen Umgang nachgewiesen wurden als bei Frauen (Scott 2011).

Das Ergebnis legt nahe, dass Männer mit aktuellen posttraumatischen Symptomen möglicherweise größere Probleme haben, damit umzugehen als allgemein häufig vom „starken Geschlecht“ angenommen wird.

Hierbei spielen jedoch im Einzelfall Kontextfaktoren wie beispielsweise soziale Unterstützung aus Familie oder Bekanntenkreis immer auch eine entscheidende Rolle.

Die Posttraumatische Belastungsstörung zeichnet sich dadurch aus, dass auf traumatische Erlebnisse, „[...] von außergewöhnlicher Bedrohung mit katastrophalem Ausmaß, [die] nahezu bei jedem tief greifende Verzweiflung auslösen würde[n]" (WHO 2005), auf besondere Weise reagiert wird. Hierzu gehört das ständige Wiedererleben des Erfahrenen in Form von wiederkehrenden Bildern (Intrusionen), Vermeidung von Gedanken oder Aktivitäten, die mit den Ereignissen verbunden werden sowie anhaltende erhöhte Erregung (Hyperarousal). Die Symptome müssen länger als einen Monat andauern (APA 2000).

\subsubsection{Sonstige Erkrankungen und Inanspruchnahme des Gesundheitssystems}

In vielen Untersuchungen wird der Zusammenhang einer Posttraumatischen Belastungssymptomatik mit anderen psychopathologischen Symptomen (Depressivität, Zwanghaftigkeit, soziale Unsicherheit, etc.) (Kuwert et al. 2008, 2009; Fischer et al. 
2006) deutlich. Insgesamt scheint eine PTBS überdurchschnittlich häufig mit medizinischen Problemen einherzugehen. So zeigten Weisberg und Kollegen (2002) den Zusammenhang zwischen Posttraumatischer Belastungsstörung und weiteren körperlichen Belastungen wie Arthritis, Asthma, Rückenschmerz, Diabetes, etc. Glaesmer und Kollegen (2011a) konnten dieses Ergebnis innerhalb einer Stichprobe der deutschen Allgemeinbevölkerung für verschiedenste kardio-vaskuläre Erkrankungen nachweisen (koronare Herzkrankheit, Angina Pectoris, Bluthochdruck, etc.). Weiter sei diese Form der Erkrankungen wie auch gastro-intestinale Symptome im Alter je eher zu finden, desto jünger der Soldat zur Zeit der Traumatisierung gewesen ist (Pizarro et al. 2006). Eine erhöhte Neigung zu suizidalen Gedanken und Suizidhandlungen ist, bislang vor allem am Beispiel von US-Soldaten, mehrfach belegt worden (Maguen et al. 2011; Kühn 2009).

Das erhöhte Risiko, aufgrund von traumatischen (Kriegs-)Erfahrungen Symptome einer posttraumatischen Belastungsstörung zu entwickeln, geht mit einem erhöhten Risiko begleitender körperlicher und psychischer Belastungen einher.

Andere Studienergebnisse belegen, dass eine aktuelle PTBS mit einer erhöhten Inanspruchnahme des Gesundheitssystems einhergeht. Es ist also anzunehmen, dass die Männer des WK II, die (noch) heute eine posttraumatische Symptomatik aufgrund der Kriegserfahrungen aufweisen, häufiger ärztliche Leistungen wegen psychischer und körperlicher Beschwerden in Anspruch nehmen sowie in stärkerem Maße hospitalisiert sind (gemessen an der Dauer von Krankenhausaufentahlten). Der Zusammenhang zwischen erlebten Traumata und PTBS auf der einen Seite und Inanspruchnahme gesundheitlicher Leistungen auf der anderen Seite wird zum Teil über körperliche Beschwerden vermittelt, bleibt jedoch bestehen, wenn diese konstant gehalten werden (Glaesmer 2011b).

Traumatisierung sowie resultierende Symptome einer Posttraumatischen Belastungsstörung bewirken zum Teil unabhängig von der Anzahl körperlicher Beeinträchtigungen ein häufigeres Aufsuchen von Ärzten und längere Krankenhausaufenthalte.

\section{2 „WK II-Männer“ heute}

\subsubsection{Chronische PTBS: Ein Leben lang Symptome?}

Von einer chronischen PTBS wird dann gesprochen, wenn das traumatische Ereignis, auf das die Symptome sich beziehen, mehr als 50 Jahre zurückliegt (Op den Velde 1990). Im Falle von vor 1946 geborenen Männern, die aktuell noch immer unter PTBS-Symptomen leiden, wäre also von einer chronischen Form der PTBS zu sprechen. Das „Überlebenden-Syndrom“, wie die chronische PTBS auch genannt wird (Sadavoy 1997), besteht also über ein ganzes Leben und zeichnet sich durch immer wiederkehrende Symptome (Angst, Affektverflachung, Depression, dysphorische 
Verstimmungen, Gedächtnisbeeinträchtigungen, somatoforme Beschwerden, Schlafstörungen etc.) aus (Aarts u. Op den Velde 1996). Die Symptomatik kann stark schwanken, das heißt mehr oder weniger ausgeprägt sein, bzw. nach langer Zeit wieder oder zum ersten Mal auftreten (Trauma-Reaktivierung). Insgesamt ist das Konzept der „chronischen PTBS“ umstritten, da bislang kaum aussagekräftige Längsschnittdaten zur PTBS vorliegen.

\subsubsection{Trauma-Reaktivierung und Re-Traumatisierung}

Ob es zur Trauma-Reaktivierung kommt, ist häufig abhängig von den Lebensumständen der Personen. Eintritt ins Rentenalter, Verlust naher Menschen oder mediale Berichterstattung (Maercker u. Mehr 2006; Hiskey et al. 2008) bewirken in vielen Fällen eine Zunahme oder gar ein „Ausbrechen“ der Symptome. Auch Abwehrmechanismen wie beispielsweise Verdrängung spielen hier oftmals eine Rolle. Weiter sind Menschen im Alter zunehmend weniger belastbar und stressresistent. Mechanismen die lange zum „Funktionieren“ des Alltags beigetragen haben, sind dann möglicherweise weniger gut anwendbar (Aarts u. Op den Velde 1996). Heuft (1999) nennt ein drohendes „Ausgeliefertsein“ im Alter als möglichen Auslöser einer Trauma-Reaktivierung.

Schreuder (1996, 2000) beschreibt aus der Erfahrung mit deutschen Kriegsveteranen des 2. Weltkriegs wiederkehrende Erinnerungen und Bilder, auch in Form von Träumen. Die Betroffenen können oder wollen sich häufig nicht mitteilen und empfinden die wiedererlebten Ängste als „Verwirrungszustände“, die möglicherweise von anderen nicht verstanden würden. Heuft (1999) beschreibt eine völlige Symptomfreiheit, besonders aber die Abwesenheit einer PTBS vor einer TraumaReaktivierung.

Um die Symptome der traumatisierten Personen zu verstehen, ist es wichtig, den individuellen Kontext, sowohl des Traumas, als auch den der aktuellen Lebenssituation zu betrachten. Damit wird deutlich, wie entscheidend das subjektive (Wieder-)Erleben einer traumatischen Situation für die Therapie der Störung ist.

Von der beschriebenen Trauma-Reaktivierung abzugrenzen ist die Re-Traumatisierung, die auf ein traumatisches Ereignis folgt, dem ein erstes erlebtes Trauma vorausgegangen ist. So käme es häufig vor, so Burgmer und Heuft (2004), dass ältere Personen nach belastenden oder gar traumatischen Ereignissen des Alltags wie beispielsweise Verkehrsunfällen oder plötzlichen körperlichen Einschnitten (z.B. Schlaganfall), vergangene Traumata reaktiviert erleben, inklusive der posttraumatischen Symptome.

Abbildung 1 bietet eine schematische Darstellung des zeitlichen Verlaufs verschiedener Traumaformen über die Lebensphase. 


$\begin{array}{cccc}\begin{array}{c}\text { Kindheits- } \\ \text { traumatisierung }\end{array} & \text { Kumulatives } & \text { Traumatisierung / } & \begin{array}{c}\text { Trauma- } \\ \text { reaktivierung }\end{array}\end{array}$

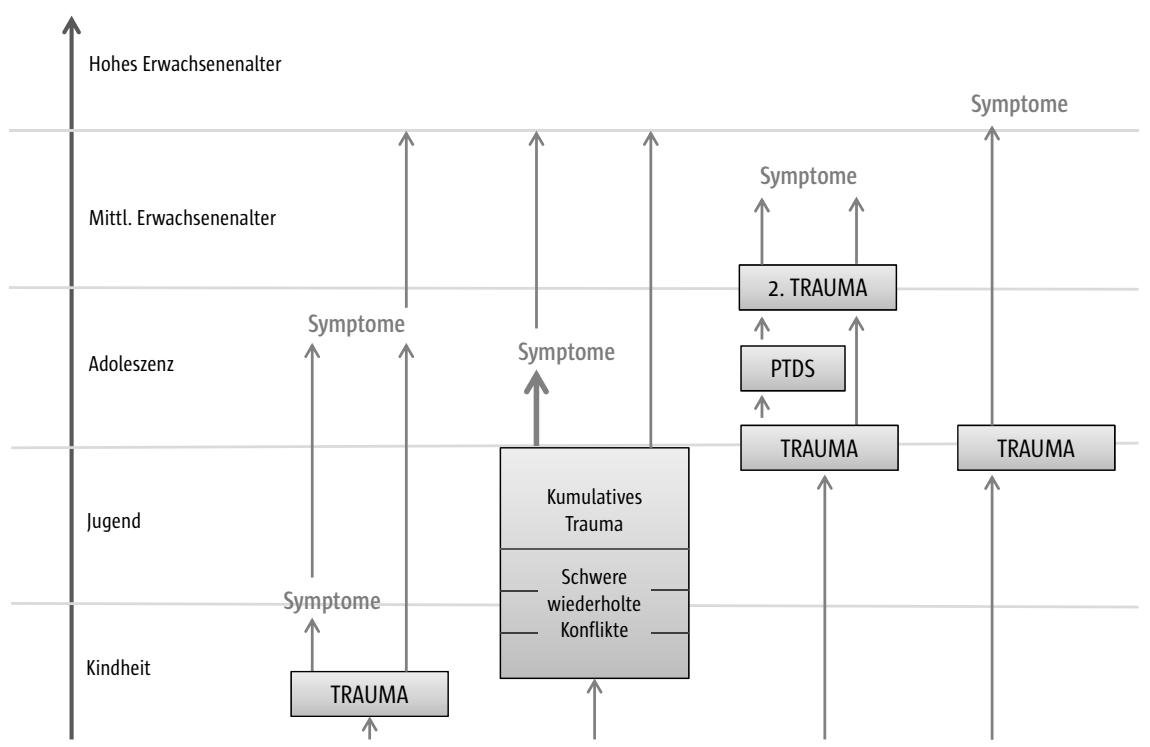

Abb. 1 Differenzielle Psychotraumatologie (Heuft 1999, mit freundlicher Genehmigung von Springer Science and Business Media)

\subsection{3 „WK II-Männer“ in Pflege und medizinischer Versorgung}

Wie bereits in den vorhergehenden Kapiteln beschrieben, ist die Symptomatik einer PTBS sehr vielschichtig.

Von lebenslang wiederkehrenden Erinnerungen eines Jahrzehnte zurückliegenden Erlebnisses bis hin zu quälenden, unerklärlichen Angstsymptomen die im hohen Alter scheinbar aus dem Nichts auftreten, ist alles möglich.

Hinzu kommt häufig Aggressivität, die durch Hilflosigkeit und Pflegebedürftigkeit im Alter hervorgerufen werden kann. Diese Aggressivität wird oft von Pflegepersonal berichtet, welches ratlos mit Wutausbrüchen oder Verweigerungen älterer Menschen umgehen muss. Aber auch Ärzte oder Familienmitglieder können Zeugen unzuordenbarer Feindseligkeit und Frustration ehemals traumatisierter Personen werden. Ursache hierfür sind häufig reaktivierte Traumatisierungen, wobei die Wut und Aggressivität Ausdruck der Hilflosigkeit in der zurückliegenden Trauma-Situation sein kann. Im Falle von Kriegsveteranen, so Heuft (1999), spielt häufig eine Verbitterung darüber eine große Rolle, dass die Kriegserlebnisse und -verletzungen gesellschaftlich zu wenig anerkannt wurden. 
Manchmal leiden die ehemaligen Soldaten bis zum Tod unter direkten oder indirekten psychischen und körperlichen Folgen von Kampfhandlungen, die als solche oft nicht zugeordnet werden.

Insgesamt ist es also wichtig, ärztliches und Pflegepersonal dahingehend zu sensibilisieren, dass Reaktionen von Patienten oder zu Pflegenden möglicherweise durch belastende Kriegserfahrungen mitbedingt werden. Hieran muss sich ein geduldiger und wertschätzender Umgang mit den entsprechenden Personen anschließen.

\section{Fazit}

Zusammenfassend lässt sich sagen, dass Kriegserleben mit Traumatisierungen einhergeht, woraus in wenigen Fällen posttraumatische Belastungssymptome resultieren; dies jedoch nachgewiesenermaßen in der Kriegsgeneration der vor 1946 geborenen Deutschen häufiger als in der restlichen Bevölkerung.

Die Tatsache, dass ein Geschlechtervergleich keine Unterschiede hinsichtlich der Entwicklung einer PTBS zeigt, bedeutet für den Mann als Kriegszeuge eine erhöhte Vulnerabilität für diese traumaassoziierten Symptome, als dies sonst unabhängig von einer Kriegsteilnahme der Fall wäre. Die mit möglichen posttraumatischen Symptomen zunehmenden psychischen und körperlichen Beschwerden schlagen sich in einem vergleichbar häufigeren Aufsuchen von Ärzten und längeren Krankenzeiten nieder, wobei die Traumatisierung per se die Inanspruchnahme medizinischer Leistungen erhöht.

Weiter können traumatisierte Personen mit posttraumatischer Symptomatik besondere Sensibilität im Umgang erforderlich machen, wenn diese zum Beispiel Behandlungen verweigern oder mit Aggression oder Feindseligkeit auf routinemäßige medizinische oder pflegerische Vorgänge reagieren.

Der Stellenwert möglicher Trauma-Reaktivierungen oder auch Re-Traumatisierungen muss langfristig ins Bewusstsein der Versorgungsstrukturen für ältere Menschen übergehen. Auch wenn dies für die Generation des 2. Weltkriegs möglicherweise spät geschieht, wird eine Sensibilität für individuelle biografische Traumata der auch nach 1946 Geborenen in neuen Situationen der Hilfsbedürftigkeit und Ohnmacht im Alter gut tun.

Letztendlich sollte die Forschung und Berichterstattung über die psychischen Folgen der vergangenen militärischen Kriegsteilnahme Anlass geben, die heutige psychologische und psychiatrische Versorgung von Soldaten der Deutschen Bundeswehr gezielt anzupassen und zu intensivieren. Dies würde beispielsweise präventive und Hemmungen abbauende Aufklärung über die Symptome einer Posttraumatischen Belastungsstörung bedeuten, ebenso wie ein flächendeckendes und niedrigschwelliges psychologisches Betreuungsangebot, von dem alle Soldaten Kenntnis haben. Die allmähliche Entwicklung einer solchen Versorgungsstruktur zeigt sich beispielsweise am geplanten Trauma-Zentrum der Bundeswehr in Berlin, dem bereits bestehenden Kompetenz-und Forschungszentrum "Fachbereich Psychische Gesundheit" am Institut für den medizinischen Arbeits- und Umweltschutz der Bundeswehr in Berlin (www.angriff-aufdie-seele.de) oder der PTBS-Hilfe-Website des Verteidungsministeriums (www.ptbs-hilfe.de). 
Letztere beinhaltet unter anderem Informationstexte für Soldaten, in denen mangelnde körperliche Fitness fatalerweise für eine mögliche PTBS mitverantwortlich gemacht wird. Aus wissenschaftlicher Sicht ist das nicht haltbar und es muss die Frage gestellt werden, ob bereits genug Wissen und Expertise innerhalb der Bundeswehr vorhanden sind, um die Entstigmatisierung von psychischen Belastungen voranzubringen.

\section{Literatur}

Aarts PG, op den Velde W (1996) Prior Traumatization and the Process of Aging. Theory and Clinical Implications. In: Van der Kolk BA, McFarlane AC \& WL, editors. Traumatic Stress: the effect of overwhelming experience on mind, body, and society. New York: Guilford Press

APA (2000) Diagnostisches und Statistisches Manual Psychischer Störungen (DSM-IV-TR). Göttingen: Hogrefe

Beutel ME, Decker 0, Brähler E (2007) Quality of life and distress among former refugees - A representative community survey of Germans born before 1946. Zeitschrift für Psychosomatische Medizin und Psychotherapie 53, 203-215

Brähler E, Decker 0, Radebold H (2004) Ausgebombt, vertrieben, vaterlos - Langzeitfolgen bei den Geburtsjahrgängen 1930-1945 in Deutschland. In: Radebold H (Hrsg.) Kindheiten im II. Weltkrieg und ihre Folgen. Gießen: Psychosozial-Verlag, 111-136

Breslau N (2009) The Epidemiology of Trauma, PTSD, and Other Posttrauma-Disorders. Trauma Violence \& Abuse 10, $198-210$

Burgmer M, Heuft $G$ (2004) Occurrence and treatment of post-traumatic stress disorder in an elderly patient after a traffic accident. International Journal of Geriatric Psychiatry 19, 185-188

Decker 0, Brähler E, Radebold H (2005) War childhood and fatherlessness - Indications for a psychosocial impact present after 15 years. Psychotherapie Psychosomatik Medizinische Psychologie 55, 96

Fairbank JA, Ebert L, Costello El (2000) Epidemiology of traumatic events and post-traumatic Stress Disorder. Diagnosis, Management and Treatment. London: Martin Dunitz.

Fischer Cl, Struwe J, Lemke MR (2006) Long-term effects of traumatic experiences on somatic and psychic complaints of German World War Two refugees. Nervenarzt 77, 58-63

Forstmeier S, Kuwert P, Spitzer C, Freyberger HJ, Maercker A (2009) Posttraumatic Growth, Social Acknowledgment as Survivors, and Sense of Coherence in Former German Child Soldiers of World War II. American Journal of Geriatric Psychiatry 17, 1030-1039

Franz M, Lieberz K, Schmitz N, Schepank H (1999) The missing father. Epidemiological findings on the significance of early absence of the father for mental health in later life. Zeitschrift fur Psychosomatische Medizin und Psychotherapie 45, 260-278

Glaesmer H, Brähler E, Gundel H, Riedel-Heller SG (2011a) The Association of Traumatic Experiences and Posttraumatic Stress Disorder With Physical Morbidity in Old Age: A German Population-Based Study. Psychosomatic Medicine 73, 401-406

Glaesmer H, Brähler E, Riedel-Heller SG, Freyberger HI, Kuwert P (2011b) The association of traumatic experiences and posttraumatic stress disorder with health care utilization in the elderly - a German population based study. General Hospital Psychiatry 33, 177-184

Glaesmer H, Gunzelmann T, Brähler E, Forstmeier S, Maercker A (2010) Traumatic experiences and post-traumatic stress disorder among elderly Germans: results of a representative population-based survey. International Psychogeriatrics 22, 661-670

Heuft G (1999) Significance of trauma reactivation in old age. Zeitschrift für Gerontologie und Geriatrie 32, 225230

Heuft G (2001) Absent fathers - Consequences of war for children in psychoanalysis. Psyche - Zeitschrift für Psychoanalyse und ihre Anwendungen 55, 1254-1256

Heuft G, Schneider G, Klaiberg A, Brähler E (2007) Bombed out - Psychic and psychosomatic long term consequences of World War II for the cohort born until 1945 in the year 2004. Zeitschrift für Psychosomatische Medizin und Psychotherapie 53, 228-243 
Hiskey S, Luckie M, Davies S, Brewin CR (2008) The phenomenology of reactivated trauma memories in older adults: A preliminary study. Aging \& Mental Health 12, 494-498

Kühn BM (2009) Soldier suicide rates continue to rise: military, scientists work to stem the tide. JAMA 301, 11111113

Kuwert P, Brähler E, Glaesmer H, Freyberger HI, Decker 0 (2009) Impact of forced displacement during World War II on the present-day mental health of the elderly: a population-based study. International Psychogeriatrics 21, 748-753

Kuwert P, Spitzer C, Dudeck M, Vogel M, Freyberger HJ, Ermann M (2008) Psychological complaints, interpersonal problems, quality of life and sense of coherence in former German warchildren. Psychotherapie Psychosomatik Medizinische Psychologie 58, 257-263

Kuwert P, Spitzer C, Träder A, Freyberger HI, Ermann M (2006) Sixty years later: post-traumatic stress symptoms and current psychopathology in former German children of World War II. International Psychogeriatrics 19, 955-961

Maercker A, Forstmeier S, Wagner B, Glaesmer H, Brähler E (2008) Post-traumatic stress disorder in Germany. Results of a nationwide epidemiological study. Nervenarzt 79, 577-586

Maercker A, Mehr A (2006) What if victims read a newspaper report about their victimization? A study on the relationship to PTSD symptoms in crime victims. European Psychologist 11, 137-142

Maguen S, Luxton DD, Skopp NA, Gahm GA, Reger MA, Metzler T), Marmar CR (2011) Killing in combat, mental health symptoms, and suicidal ideation in Iraq war veterans. Journal of Anxiety Disorders 25, 563-567

Op den Velde W (1990) Current psychiatric complaints of Dutch Resistance Veterans: A feasability study. Journal of Traumatic Stress 3, 351-358

Pizarro J, Silver RC, Prause I (2006) Physical and mental health costs of traumatic war experiences among civil war veterans. Archives of General Psychiatry 63, 193-200

Sadavoy I (1997) Survivors: A review of the late-life effects of prior psychological trauma. American Journal of Geriatric Psychiatry 5, 287-301

Schreuder IN (1996) Posttraumatic re-experiencing in older people: Working through or covering up? American Journal of Psychotherapy 50, 231-242

Schreuder JN, Kleijn WC, Rooijmans HGM (2000) Nocturnal re-experiencing more than forty years after war trauma. Journal of Traumatic Stress 13, 453-463

Scott KM (2011) Sex differences in the disability associated with mental disorders. Current Opinion in Psychiatry 24, 331-335

Seikowski K, Paasch U (2010) Der alternde Mann. In: Bardehle D, Stiehler M, editors. Erster Deutscher Männergesundheitsbericht. Germering/München: Zuckerschwerdt Verlag. 58-70

Spitzer C, Barnow S, Volzke H, John U, Freyberger HJ, Grabe HJ (2008) Trauma and posttraumatic stress disorder in the elderly: Findings from a German community study. Journal of Clinical Psychiatry 69, 693-700

Weisberg RB, Bruce SE, Machan JT, Kessler RC, Culpepper L, Keller MB (2002) Nonpsychiatric illness among primary care patients with trauma histories and posttraumatic stress disorder. Psychiatric Services 53, 848-854

WHO (2005) Internationale Klassifikation Psychischer Störungen: ICD-10, Kapitel V(F). Klinisch-diagnostische Leitlinien. Bern: Verlag Hans Huber

Zlotnick C, Rodriguez BF, Weisberg RB, Bruce SE, Spencer MA Culpepper L, Keller MB (2004) Chronicity in posttraumatic stress disorder and predictors of the course of posttraumatic stress disorder among primary care patients. Journal of Nervous and Mental Disease 192, 153-159 